\title{
Refractive index and its impact on pseudophakic dysphotopsia
}

\author{
Bryce R Radmall' \\ Anne Floyd ${ }^{2}$ \\ Zack Oakey ${ }^{3}$ \\ Randall J Olson ${ }^{4}$ \\ 'Department of Ophthalmology, \\ Casey Eye Institute, Oregon Health \\ and Science University, Portland, \\ OR, ${ }^{2}$ Department of Ophthalmology, \\ Louisiana State University Health \\ Sciences Center, Shreveport, LA, \\ ${ }^{3}$ Department of Ophthalmology, Gavin \\ Herbert Eye Institute, University \\ of California, Irvine, CA, ${ }^{4}$ Department \\ of Ophthalmology and Visual Sciences, \\ John A. Moran Eye Center, University \\ of Utah, Salt Lake City, UT, USA
}

Correspondence: Randall J Olson John A. Moran Eye Center, Department of Ophthalmology, University of Utah, 65 Mario Capecchi Drive,

Salt Lake City, UT 84I32, USA

$\mathrm{Tel}+\mathrm{I} 8015856622$

Fax + I 80I 58I 3357

Email randallj.olson@hsc.utah.edu
This article was published in the following Dove Press journal:

Clinical Ophthalmology

20 July 2015

Number of times this article has been viewed

Purpose: It has been shown that the biggest dissatisfier for uncomplicated cataract surgery patients is pseudophakic dysphotopsia (PD). While edge design of an intraocular lens (IOL) impacts this problem, refractive index is still controversial as to its impact. This retrospective cohort study was designed to determine the role of increasing refractive index in PD.

Patients and methods: This study was conducted at the John A. Moran Eye Center, University of Utah, USA. A retrospective chart review identified patients who received one of two hydrophobic acrylic single piece IOLs (AcrySof WF SP [SN60WF] or Tecnis SP [ZCB00]), which differed mainly by refractive index (1.55 versus 1.47$)$. Eighty-seven patients who had received implantation of a one-piece hydrophobic acrylic IOL were enrolled. Patients were included if the surgery had been uncomplicated and took place at least a year before study participation. All eligible patients had 20/20 best corrected vision, without any disease known to impact visual quality. In addition to conducting a record review, the enrolled patients were surveyed for PD, using a modified National Eye Institute Visual Function questionnaire, as well as for overall satisfaction with visual quality.

Results: Statistical analysis demonstrated no difference between the two cohorts regarding PD, general visual function, and overall visual satisfaction.

Conclusion: The study suggests that with the two IOLs assessed, increasing the refractive index does not increase incidence of PD or decrease overall visual satisfaction.

Keywords: cataract, phacoemulsification, refractive index, visual function, patient satisfaction

\section{Introduction}

As phacoemulsification cataract surgery has improved outcomes and patient satisfaction, patients with excellent vision report that they remain unhappy despite uncomplicated surgical results. Previous studies have shown that this is largely due to pseudophakic dysphotopsia (PD). ${ }^{1,2}$ PD is caused by non-physiological scattering or blockage of light induced by intraocular lenses (IOLs). Indeed, both previous studies have clearly shown that PD is the only significant correlate with dissatisfaction and visual dysfunction in otherwise uncomplicated patients, although their surgeon would consider the outcome to be good. It was also shown that age-matched phakic patients with good vision do not experience most of the typical PD symptoms. ${ }^{1}$ This stands to reason, as PD is caused by non-physiological refracted and reflected light introduced by the IOL implant. Refracted and reflected light results in unwanted secondary light or dark visual images.

One inherent challenge in studying PD is how to define and survey PD in a validated format. A PD survey created for a recent analysis of normal pseudophakic patients in a supernormal population was validated by its strong correlation with the National 
Eye Institute Visual Function Questionnaire, and has resulted in the optimization of a survey to study PD in a validated format. ${ }^{2}$ Thus, the pieces of the puzzle are now in place to best answer the issue of refractive index impact on PD.

This study evaluates the significance of the IOL refractive index in increasing PD symptoms, which has not been carefully investigated in the clinical setting. Theoretically, increasing refractive index should increase both the signal strength and the symptoms of PD. ${ }^{3}$

\section{Methods}

This was a retrospective case review and survey of patients who underwent uneventful phacoemulsification and IOL insertion for cataracts at the John A. Moran Eye Center, University of Utah School of Medicine. The study and data accumulation were carried out with approval from the University of Utah Institutional Review Board. The study is in accordance with Health Insurance Portability and Accountability Act (HIPAA) regulations. Informed consent for the research was obtained from all patients prior to participation.

Retrospective review of the Moran Eye Center surgery logs and corresponding patient medical records determined eligibility for survey administration. Patients who were selected had received implantation of a one-piece hydrophobic acrylic IOL, either the AcrySof ${ }^{\circledR}$ IQ WF Single-Piece (SN60WF; Alcon Laboratories, Inc., Fort Worth, TX, USA) or the Tecnis ${ }^{\circledR}$ SP (ZCB00; Abbott Medical Optics Inc, Santa Ana, CA, USA). Both of these lenses were chosen because they corrected spherical aberration, had very similar overall and edge designs, and mainly differed from each other by refractive index ( 1.55 versus 1.47 , respectively). A difference that might impact the results is the lenticular nature of higher refractive powers with the $\mathrm{ZCB} 00$ such that the optical zone does not extend to the IOL edge in IOL powers above 21 diopters; however, they are more similar than dissimilar in all other respects.

Study participation involved completion of three validated questionnaires regarding PD and visual satisfaction: our shortened and modified National Eye Institute Visual Function (NEI-VF) and PD surveys, and a Global Satisfaction survey (Figure 1).

Selection criteria included uncomplicated cataract surgery, 50 years of age or greater, best corrected visual acuity (BCVA) of 20/20 or better as assessed by a Snellen eye chart, at least 1 year since surgery so that only chronic PD symptoms were studied, and an eye exam no less than 4 months from the time of the survey. Exclusion criteria (identified by diagnosis or any mention of slit-lamp examination in the medical record) included retinal, optic nerve or corneal pathology that impacted vision (other than mild guttata or minimal hard drusen without retinal pigment epithelial pathology). Patients with post-surgical capsular opacification, diabetic retinopathy, glaucoma or chronic dry eye syndrome were also excluded. Previous history of corneal refractive procedures also disqualified patients from study participation.

The selected patients were contacted by mail and invited to participate in the study. Follow-up was performed by phone, and patients reported their responses to the three completed questionnaires. Only one eye per patient was included in the study. If patients had bilateral qualifying eyes then the better seeing or dominant eye was chosen. Some subjects had a lens other than one of the designated lenses in the non-selected eye. These patients were included, as the patient reported on the selected eye only. Patients were asked to respond for only the selected eye and were reminded to do so throughout the phone interview.

Statistical analyses were performed using SAS system V9.3 (SAS Institute Inc., Cary, NC, USA). Spearman correlations were used for comparing means between the two IOL group types. $P<0.05$ was considered statistically significant. Differences in the distribution and medians of the responses for the two IOL group types were evaluated by the Wilcoxon rank sum test.

\section{Results}

A total of 1,261 patient records were reviewed, and 113 charts met the criteria for enrollment in the study. All enrolled patients underwent surgery between 1 and 2 years prior to enrollment. Eighty-seven individuals completed the survey, with 50 in the SN60WF cohort and 37 in the ZCB00 cohort. With regard to $\mathrm{PD}$, general visual function, and overall visual satisfaction, the two IOL groups showed no significant differences (Table 1).

\section{Discussion}

PD is a disorder produced from non-physiological photic phenomena, such as scattered light created as light passes through the prosthetic lens. These non-useful and unwanted patterns of light projected onto the retinal surface may result in either positive or negative symptoms. ${ }^{2,4}$ Positive dysphotopsia stems from addition of light that produces artifacts projected onto the retina that patients may describe as glare, arcs, streaks, rings and halos. Conversely, negative dysphotopsia is thought to be produced by blockage of light from reaching certain portions of the retina. Patients describe this phenomenon as 
A. Do you have difficulty, even with glasses...

(circle answer choice)

1. Reading small print, such as labels on medicine bottles, a telephone book, food

0 . No

1. A little

2. A moderate amount

3. A great deal

4. Unable to do the activity

2. Reading a newspaper or a book
0 . No
1. A little
2. A moderate amount
3. A great deal
4. Unable to do the activity

3. Reading traffic signs, street signs, or store signs
0 . No
1. A little
2. A moderate amount
3. A great deal
4. Unable to do the activity

4. Doing fine handwork like sewing, knitting, crocheting, carpentry
0 . No
1. A little
2. A moderate amount
3. A great deal
4. Unable to do the activity

5. Writing checks or filling out forms
0 . No
1. A little
2. A moderate amount
3. A great deal
4. Unable to do the activity

B. With this survey we ask you to rate your satisfaction with your vision since your cataract surgery when considering several different scenarios that some patients have considered as problematic. When considering each situation, please consider the issue only as it relates to the study eye that we will examine. Please score each question from 0 (I have noted no problem like this or in this situation) to 10 (I find this situation debilitating). Any number in-between can be used to grade your response between these two extremes.

I. Since my surgery, oncoming headlights at night (whether you drive at night or not) have been a problem. - (score 0 [no problem] to 10 [debilitating])

2. Since my surgery, I am bothered by halos around bright lights. - (score 0 [no problem] to 10 [debilitating])

3. Since my surgery, I have noted a dark or gray shadow to the side of my vision. - (score 0 [no problem] to 10 [debilitating])

4. Since my surgery, bright lights off to the side or above me are annoying. - (score 0 [no problem] to 10 [debilitating])

5. Since my surgery, when looking at lights, I notice a flickering shadow that moves from side to side of the light. - (score 0 [no problem] to 10 [debilitating])

6. Since my surgery, I have noted a semi-circular shadow in my vision. - (score 0 [no problem] to 10 [debilitating])

C. My overall satisfaction with my vision since my surgery: please rate your overall satisfaction with the vision in the eye since your cataract surgery that is part of this study from 0 (totally unsatisfied) to 10 (totally satisfied). -

Figure I The three surveys administered to study participants.

Notes: (A) The Modified National Eye Institute Visual Function questionnaire. (B) The Pseudophakic Dysphotopsia Scale questionnaire. (C) The one-question Global Satisfaction survey.

shadows, dark spots, or crescents typically perceived in the temporal visual field. A recent study suggests that negative dysphotopsia is created when parallel rays of light passing at the nasal, posterior edge of a truncated IOL results in two divergent rays of light that create a shadow or penumbra that may be perceived in the temporal visual field. ${ }^{5,6}$ Another study previously had suggested that negative dysphotopsia symptoms directly correlated with the amount of anterior capsule coverage of the IOL. ${ }^{7}$ however, Kinard et al indicated that the degree of overlap between the anterior capsule and IOL implant had little bearing on the creation of dysphotopsias; thus, evaluation of the capsulotomy was not

Table I Descriptive statistics by lens type and overall

\begin{tabular}{|c|c|c|c|c|c|c|c|c|c|c|}
\hline & \multicolumn{3}{|l|}{ SN60WF } & \multicolumn{3}{|l|}{ ZCBO0 } & \multicolumn{3}{|l|}{ All } & \multirow[t]{3}{*}{$\boldsymbol{P}$-value* } \\
\hline & \multicolumn{3}{|l|}{$\mathbf{N}=\mathbf{5 0}$} & \multicolumn{3}{|l|}{$\mathbf{N}=\mathbf{3 7}$} & \multicolumn{3}{|l|}{$\mathbf{N}=87$} & \\
\hline & Mean \pm SD & Median & Range & Mean \pm SD & Median & Range & Mean \pm SD & Median & Range & \\
\hline Global Satisfaction I item & $9.23 \pm 0.88$ & 9.5 & $7-10$ & $9.07 \pm \mid .31$ & 10 & $4-10$ & $9.16 \pm 1.08$ & 10 & $4-10$ & 0.889 \\
\hline NEI-VF Scale 5 items & $0.98 \pm 1.62$ & 0 & $0-8$ & $1.35 \pm 2.45$ & 0 & $0-9$ & $1.14 \pm 2.01$ & 0 & $0-9$ & 0.958 \\
\hline PD Scale & $2.98 \pm 4.8$ & 0 & $0-18$ & $3.57 \pm 5.04$ & 2 & $0-25$ & $3.23 \pm 4.88$ & 1 & $0-25$ & 0.172 \\
\hline
\end{tabular}

Notes: *P-value is for the Wilcoxon rank sum test for differences in the distribution and medians in the two lens groups. SN60WF, AcrySof ${ }^{\circledR}$ IQ WF Single-Piece (Alcon Laboratories Inc., Fort Worth, TX, USA). ZCB00, Tecnis ${ }^{\circledR}$ SP (Abbott Medical Optics Inc, Santa Ana, CA, USA).

Abbreviations: NEI-VF, National Eye Institute Visual Function; PD, pseudophakic dysphotopsia; SD, standard deviation. 
directly evaluated in this study. Both positive and negative dysphotopsias are often transient and frequently present only in certain lighting conditions or positioning. To some surgeons, patients' description of their visual complaint seems surprising, given that the clinician will often measure the visual acuity to be 20/20 and yet dysphotopsia will be present. These two occurrences are independent of each other. ${ }^{1,2,8}$

Despite the resounding success of modern phacoemulsification cataract surgery in terms of visual acuity, the prevalence of the "20/20 dissatisfied patient" remains a tangible entity in ophthalmic practices throughout the world. Although a wide array of variables may be contributory, PD is a well-known and important source of visual dissatisfaction. As such, it certainly deserves increased attention in order to help ameliorate this problem. Currently, the ophthalmic industry spends enormous resources in the perfection of preoperative biometry or determination of accurate cylinder and sphere measurements; yet it has been shown that the degree of PD is actually what optimally correlates with a patient's overall visual satisfaction and function in uncomplicated surgical cases without confounding pathology and with good visual acuity.,

Imperfections in the optical properties of the IOL implant have been implicated in the production of dysphotopsias. ${ }^{4}$ Others have assessed aspects of lens properties and design that contribute to the production of PD. ${ }^{3,5,6,9-14}$ Various studies have demonstrated that IOL edge design certainly impacts the problem. ${ }^{3,5,6,12-14}$ Another property of IOL design, specifically refractive index, remains controversial as to its impact on the formation of PDs. One study in particular strongly suggested that increasing refractive index increases PD. ${ }^{13}$ As with many extant publications on the subject, this previous study suffered from confounders that could impact patient satisfaction reports.

This study examined PD and how it correlated with visual satisfaction and function in patients who previously had undergone uncomplicated cataract surgery, using one of two study IOLs that mainly differed by refractive index. Both the SN60WF and the ZCB00 cohorts had documented excellent outcomes as measured by BCVA, and both cohorts were screened for any confounding diseases or surgical complications to the extent that this is possible in a chart review. We sought to demonstrate the difference, if any, in the incidence of PD complaints and satisfaction in patients with a higher refractive index IOL (1.55) versus those with a lower refractive index IOL (1.47).

Though other IOL parameters such as overall and edge design are known to impact the problem of $\mathrm{PD}$, the impact of refractive index has remained controversial. This had been poorly studied to date in a clinical setting, although a study which looked at laser ray tracing indicated that the problem should positively correlate with refractive index. ${ }^{3}$

Through careful exclusion of confounding diseases, our record review of nearly 1,300 pseudophakic patients resulted in the creation of two study cohorts that mainly differed by the refractive index of the IOL implant. Each of the three study surveys was designed to assess a discrete aspect of visual quality. The first survey, a truncated version of the NEI-VF questionnaire, targeted day-to-day visual function. The second survey, known as the Pseudophakic Dysphotopsia Scale, focused on common forms of positive and negative dysphotopsia complaints. In the final survey the patients ranked their overall satisfaction with their vision on a scale from 0 to 10, with 10 being highly satisfied. Analysis of the data collected from these previously validated surveys has resulted in a clear answer to our clinical question: at least in regard to the two studied IOLs, increasing refractive index did not result in an increased incidence of PD or functional complaints. Furthermore, this study did not show a statistical difference in the reported visual function or overall satisfaction between the high and the low refractive index study groups.

While a previous study suggested that a high refractive index IOL might have a greater propensity for producing $\mathrm{PD},{ }^{13}$ we were unable to replicate such a correlation with our study population to the extent that this could be determined from a retrospective chart review.

As would be expected in a population selected for excellent visual acuity without significant concomitant ocular disease, the satisfaction scores were high. On a scale of $0-10$, with 0 being totally dissatisfied and 10 being totally satisfied, together, the two cohorts averaged a mean of $9.16 \pm 1.08$, indicating a very high level of satisfaction. Both lenses performed well in terms of visual function and resulted in equally strong overall satisfaction scores. The equivalent performance by the study lenses likely is a reflection of our finding that no significant difference could be detected with regard to $\mathrm{PD}$.

While we did not find a correlation of refractive index with PD complaints, additional studies should be conducted to determine what aspects of IOL design should be considered when selecting a prosthetic implant, so as to minimize the PD impact. Comparative studies similar to this one should investigate the impact that IOL shape, material, and edge design have on PD. In so doing, one would hope to minimize the impact of PD in post-cataract patients, and to find clues 
as to what design characteristics or surgical variations are needed to minimize this problem.

It is important to note although all three surveys showed no statistical difference between the two study IOLs, a future evaluation of our findings regarding the Dysphotopsia Scale ( $P=0.172$ ) would benefit from inclusion of a larger population than the cohort we evaluated. Increasing the number of subjects would not affect overall satisfaction results $(P=0.889)$ or the NEI-VF results $(P=0.958)$. However, assuming a similar probability density and standard deviation, the difference observed in the PD scale may become significant at $P \leq 0.05$ with a relatively small (approximately ten) increase in the number of cases. The difference in such case would be in favor of the higher refractive index IOL, however.

A major weakness of this study is that the two IOLs studied are not exact replicas of one another, except for the difference in refractive index. Differences include edge design, optical zone size, presence of a blue blocking chromophore, and tendency for glistenings. Consequently, potential confounders resulting from the design of the study IOLs may obscure the PD picture. Given the multiplicity of IOL shapes and design, it remains impossible to select two IOLs that differ solely by refractive index. We controlled for variance in IOL design to the best extent possible by selecting two IOLs that both correct for spherical aberration and have similar overall and edge design except for refractive index.

The high satisfaction scores clearly were related to absence of PD across both groups; consequently, our study findings were limited due to a reduced power to detect a difference in PD. Furthermore, due to the nature of retrospective chart reviews, it was not feasible to eliminate all potential confounding ocular conditions and perhaps to adequately consider other clinical details that may have been important in the status of the enrolled subjects. Nonetheless, we believe that our two study cohorts had minimal confounding variables represented, as we developed stringent inclusion and exclusion criteria at least equivalent to those of similar studies of PD. ${ }^{1,2,13}$

In conclusion, we show that increasing refractive index with these two IOLs did not increase the incidence of PD, nor did it decrease overall visual satisfaction. Selecting the best IOL for cataract surgery based on the degree of refractive index appears to be of minimal importance in optimizing postsurgical visual outcomes, at least with these two IOLs.

\section{Acknowledgments}

Molly McFadden, MS, Division of Epidemiology, Department of Internal Medicine, University of Utah, provided statistical analysis of the data. Susan Schulman, MAT, School of Medicine, University of Utah, provided writing and editing assistance. This research was presented at the 2013 ASCRS meeting, San Francisco, CA, USA.

\section{Financial and material support}

This study was supported in part by an unrestricted grant from Research to Prevent Blindness, Inc., New York, New York, USA, to the Department of Ophthalmology and Visual Sciences, University of Utah, Salt Lake City, Utah, USA. This investigation also was supported in part by the University of Utah Study Design and Biostatistics Center, with funding in part from the National Center for Research Resources and the National Center for Advancing Translational Sciences, National Institutes of Health, through Grant 8UL1TR000105 (formerly UL1RR025764).

\section{Disclosure}

None of the authors have any financial or proprietary interests in any product, material or method mentioned nor any other conflict of interest to report.

\section{References}

1. Welch NR, Gregori N, Zabriskie N, Olson RJ. Satisfaction and dysphotopsia in the pseudophakic patient. Can J Ophthalmol. 2010;45(2): 140-143.

2. Kinard K, Jarstad A, Olson RJ. Correlation of visual quality with satisfaction and function in a normal cohort of pseudophakic patients J Cataract Refract Surg. 2013;39(4):590-597.

3. Holladay JT, Lang A, Portney V. Analysis of edge glare phenomena in intraocular lens edge designs. J Cataract Refract Surg. 1999; 25(6):748-752.

4. Schwiegerling J. Recent developments in pseudophakic dysphotopsia Curr Opin Ophthalmol. 2006;17(1):27-30.

5. Holladay JT, Zhao H, Reisin CR. Negative dysphotopsia: the enigmatic penumbra. J Cataract Refract Surg. 2012;38(7):1251-1265.

6. Holladay JT. Reply: Etiology of negative dysphotopsia. J Cataract Refract Surg. 2013;39(3):486.e1-e4.

7. Masket S, Fram NR. Pseudophakic negative dysphotopsia: Surgical management and new theory of etiology. $J$ Cataract Refract Surg. 2011;37(7):1199-1207.

8. Van Den Berg TJ, Van Rijn LJ, Michael R, et al. Straylight effects with aging and lens extraction. Am J Ophthalmol. 2007;144(3):358-363.

9. Trattler WB, Whitsett JC, Simone PA. Negative dysphotopsia after intraocular lens implantation irrespective of design and material. $J$ Cataract Refract Surg. 2005;31(4):841-845.

10. Tester R, Pace NL, Samore M, Olson RJ. Dysphotopsia in phakic and pseudophakic patients: incidence and relation to intraocular lens type(2). $J$ Cataract Refract Surg. 2000;26(6):810-816.

11. Tognetto D, Toto L, Sanguinetti G, Ravalico G. Glistenings in foldable intraocular lenses. J Cataract Refract Surg. 2002;28(7):1211-1216.

12. Farbowitz MA, Zabriskie NA, Crandall AS, Olson RJ, Miller KM. Visual complaints associated with the AcrySof acrylic intraocular lens(1). J Cataract Refract Surg. 2000;26(9):1339-1345.

13. Jin $\mathrm{Y}$, Zabriskie N, Olson RJ. Dysphotopsia outcomes analysis of two truncated acrylic 6.0-mm intraocular optic lenses. Ophthalmologica. 2009;223(1):47-51.

14. Erie JC, Bandhauer MH, McLaren JW. Analysis of postoperative glare and intraocular lens design. J Cataract Refract Surg. 2001;27(4): 614-621. 


\section{Publish your work in this journal}

Clinical Ophthalmology is an international, peer-reviewed journal covering all subspecialties within ophthalmology. Key topics include: Optometry; Visual science; Pharmacology and drug therapy in eye diseases; Basic Sciences; Primary and Secondary eye care; Patient Safety and Quality of Care Improvements. This journal is indexed on

Submit your manuscript here: http://www.dovepress.com/clinical-ophthalmology-journal
PubMed Central and CAS, and is the official journal of The Society of Clinical Ophthalmology (SCO). The manuscript management system is completely online and includes a very quick and fair peer-review system, which is all easy to use. Visit http://www.dovepress.com/ testimonials.php to read real quotes from published authors. 